\title{
Effets du génotype, de l'âge et de la saison sur les composantes de la reproduction chez la Lapine (1)
}

\author{
Françoise HULOT \& G. MATHERON \\ I.N.R.A., Station d'Amélioration génétique des Animaux, \\ Centre de Recherches de Toulouse, B.P. 12, F 31320 Castanet-Tolosan
}

\begin{abstract}
Résumé
Une comparaison de 120 lapines des deux génotypes Californiens et Néo-Zélandais blancs, sélectionnées à la Station d'Amélioration génétique des animaux de Toulouse, abattues après saillies aux stades nullipare, primipare et multipare (après la troisième mise-bas) et cela aux quatre saisons de l'année, conduit aux conclusions suivantes:

- une femelle Californienne, quels que soient son âge et la saison de mesure, pond en moyenne deux ovules de plus qu'une Néo-Zélandaise blanche;

- l'étude de la viabilité pré-implantatoire donne un classement inverse de ces deux types génétiques;

- lorsque l'âge d'une femelle augmente, son niveau ovulatoire augmente tandis que sa capacité utérine aurait tendance à diminuer ;

- c'est au stade primipare qu'il y a le plus d'embryons suite à une conjonction favorable des deux mécanismes antagonistes;

- l'effet de la saison n'est pas homogène selon le caractère considéré.
\end{abstract}

\section{Introduction}

Les performances reproductives sont le facteur essentiel de la réussite économique d'un élevage de lapins, espèce prolifique à rythme de reproduction intensif. L'amélioration d'un caractère aussi complexe que la taille de portée, qui dépend notamment de l'expression du gènome d'au moins deux générations (ovulation et qualité utérine de la mère, viabilité du jeune) repose avant tout sur la connaissance des facteurs qui l'influence.

De nombreux critères modifient la productivité numérique potentielle d'une lapine. Parmi ceux-ci, le type génétique (F. Hulot \& Matheron, 1979 ; Matheron \& Poujardieu, 1976), la saison de reproduction (Selme \& Prud'hon, 1973 ; Sittman et al., 1964) et l'âge de l'animal (AdAMS, 1970 ; BigGers et al., 1962 ; Fox, 1980) ont été étudiés soit en considérant la taille de portée (caractère synthétique) soit en prenant en compte ses composantes (ovulation, mortalité embryonnaire). Nos récents travaux

(1) ATP 654-159 - Physiologie périnatale. 
(F. Hulot \& Matheron, 1979) ont montré dans le cas de saillies post-partum, la part des effets génétiques pour deux souches sélectionnées par l'I.N.R.A. dans le cadre du schéma national d'amélioration génétique du Lapin de chair, sur le nombre d'ovules pondus et la viabilité embryonnaire. De plus, nous avons pu mettre en évidence l'importance relative des voies d'action directes et indirectes (maternelle et grand maternelle) des gènes caractérisant ainsi sur ce plan ces deux souches I.N.R.A. 1066 (origine Californienne) et I.N.R.A. 1077 (origine Néo-Zélandaise).

Le but de cette présente étude est de préciser les différences globales, indépendamment de leurs voies d'action, pour ces deux types génétiques, par l'analyse de l'effet de l'âge de la femelle et de la saison de mesure sur les composantes de la productivité numérique. Ce travail doit en outre permettre de cerner plus précisément l'évolution de la capacité reproductive d'une femelle au cours de sa vie et de déterminer le moment où il faut faire les contrôles de production (âge et saison) pour l'appréhender au mieux.

\section{Matériel et méthodes}

\section{1. - Protocole expérimental}

Pour chaque génotype, 6 mâles adultes et environ 120 femelles ont été mis en cages individuelles à l'âge de 77 jours entre le 2 juin 1977 et le 11 septembre 1978 dans un bâtiment d'élevage maintenu à une température d'environ $18^{\circ} \mathrm{C}$ et éclairé en moyenne $16 \mathrm{~h}$ sur 24 heures. Les animaux sont nourris avec un granulé complet du. commerce, à 17 p. 100 de protéines et rationnés à $150 \mathrm{~g}$ par jour.

Les animaux ont été répartis en lots correspondant à trois stades de la vie reproductive d'une femelle jusqu'à sa maturité sexuelle : nullipare, primipare, multipare (dans ce dernier lot, les lapines ont toutes réalisé trois portées). Les femelles nullipares sont présentées au mâle à l'âge de $150 \pm 10$ jours. Les lapines ayant donné naissance à une portée sont ressaillies allaitantes, 10 ou 11 jours après la mise-bas. Tous les accouplements sont réalisés en race pure à jour fixe de la semaine. Les contrôles biologiques concernant la première portée (femelles nullipares), la deuxième portée (femelles primipares) et la quatrième portée (femelles multipares) sont effectués 12 jours après la saillie en prélevant le tractus génital après laparatomie. Les informations concernant l'ovulation (nombre de corps jaunes), et la mortalité embryonnaire (nombre de sites d'implantation et d'embryons) sont enregistrées, après un comptage à l'œil nu, éventuellement suivi d'un contrôle à la loupe binoculaire dans les cas litigieux (dissection de corps jaunes en particulier).

La planification de l'expérience a été conçue de telle manière que les trois lots correspondant aux stades de parités étudiés, soient synchrones au moment de la laparatomie. Les examens furent groupés sur deux semaines pour les femelles nullipares et primipares et étalés sur un mois pour les femelles multipares.

Afin de répartir de façon aléatoire l'influence du niveau génétique des femelles d'une même souche et celle de l'effet maternel (notamment stade physiologique des mères des lapines mises en expérience) vis-à-vis des estimations des effets pris en compte dans le modèle, nous avons mis en place dans la mesure du possible des 
demi-sœurs de père aux différentes combinaisons saison-stade du protocole, et des femelles pleines sœurs issues de mères de même âge, aux différents stades. Cet ensemble expérimental a été réalisé quatre fois dans l'année afin de contrôler l'influence saisonnière, la moyenne des opérations se situant les 15 février, 15 mai, 15 août et 15 novembre 1978. Nous avons regroupé les résultats dans 4 lots correspondant aux saisons calendaires. 112 femelles au total ont ainsi été prélevées, les divers effectifs par cellule élémentaire étant indiqués au tableau 1.

\section{TABleau 1}

Effectifs des femelles saillies (1) ovulant (2) et avec des sites (3)

Number of mated females and of females with ovulation and implantation sites

\begin{tabular}{|c|c|c|c|c|c|c|c|}
\hline Parité $\quad$ Saison & Races & $\mathbf{H}$ & $\mathrm{P}$ & $\mathrm{E}$ & A & $\begin{array}{l}\text { Totaux } \\
\text { partiels }\end{array}$ & Totaux \\
\hline \multirow{2}{*}{ Nullipares } & Cal. & $\begin{array}{ll}7 & (1) \\
3 & (2) \\
2 & (3)\end{array}$ & $\begin{array}{l}6 \\
6 \\
5\end{array}$ & $\begin{array}{l}5 \\
5 \\
4\end{array}$ & $\begin{array}{l}8 \\
7 \\
7\end{array}$ & $\begin{array}{l}26 \\
21 \\
18\end{array}$ & $\begin{array}{l}56(1) \\
43(2)\end{array}$ \\
\hline & N.-Z. & $\begin{array}{l}7 \\
3 \\
3\end{array}$ & $\begin{array}{l}8 \\
6 \\
5\end{array}$ & $\begin{array}{l}8 \\
7 \\
6\end{array}$ & $\begin{array}{l}7 \\
6 \\
6\end{array}$ & $\begin{array}{l}30 \\
22 \\
20\end{array}$ & $38(3)$ \\
\hline \multirow{2}{*}{ Primipares } & Cal. & $\begin{array}{l}8 \\
3 \\
1\end{array}$ & $\begin{array}{l}8 \\
5 \\
5\end{array}$ & $\begin{array}{l}6 \\
3 \\
2\end{array}$ & $\begin{array}{l}9 \\
5 \\
4\end{array}$ & $\begin{array}{l}31 \\
16 \\
12\end{array}$ & $\begin{array}{l}62 \\
35\end{array}$ \\
\hline & N.-Z. & $\begin{array}{l}8 \\
7 \\
6\end{array}$ & $\begin{array}{l}8 \\
2 \\
2\end{array}$ & $\begin{array}{l}7 \\
6 \\
5\end{array}$ & $\begin{array}{l}8 \\
4 \\
4\end{array}$ & $\begin{array}{l}31 \\
19 \\
17\end{array}$ & 29 \\
\hline \multirow{2}{*}{ Multipares } & Cal. & $\begin{array}{l}6 \\
4 \\
3\end{array}$ & $\begin{array}{r}12 \\
8 \\
5\end{array}$ & $\begin{array}{l}7 \\
4 \\
3\end{array}$ & $\begin{array}{l}7 \\
3 \\
3\end{array}$ & $\begin{array}{l}32 \\
19 \\
14\end{array}$ & \\
\hline & N.-Z. & $\begin{array}{l}9 \\
3 \\
3\end{array}$ & $\begin{array}{l}7 \\
5 \\
5\end{array}$ & $\begin{array}{l}8 \\
5 \\
4\end{array}$ & $\begin{array}{l}4 \\
2 \\
2\end{array}$ & $\begin{array}{l}28 \\
15 \\
14\end{array}$ & 60 \\
\hline \multirow{2}{*}{$\begin{array}{l}\text { Totaux } \\
\text { partiels }\end{array}$} & Cal. & $\begin{array}{r}21 \\
10 \\
6\end{array}$ & $\begin{array}{l}26 \\
19 \\
15\end{array}$ & $\begin{array}{r}18 \\
12 \\
9\end{array}$ & $\begin{array}{l}24 \\
15 \\
14\end{array}$ & $\begin{array}{l}89 \\
56 \\
44\end{array}$ & $\begin{array}{l}34 \\
28\end{array}$ \\
\hline & N.-Z. & $\begin{array}{l}24 \\
13 \\
12\end{array}$ & $\begin{array}{l}23 \\
13 \\
12\end{array}$ & $\begin{array}{l}23 \\
18 \\
15\end{array}$ & $\begin{array}{l}19 \\
12 \\
12\end{array}$ & $\begin{array}{l}89 \\
56 \\
51\end{array}$ & \\
\hline \multicolumn{2}{|l|}{ Totaux } & $\begin{array}{l}45 \\
23 \\
18\end{array}$ & $\begin{array}{l}49 \\
32 \\
27\end{array}$ & $\begin{array}{l}41 \\
30 \\
24\end{array}$ & $\begin{array}{l}43 \\
27 \\
26\end{array}$ & & $\begin{array}{r}178 \\
112 \\
95\end{array}$ \\
\hline
\end{tabular}

(1) Saillies.

(2) Ovulant.

(3) A sites (1 seule femelle NZ-primipare saison A, a eu des sites sans embryons).

H: Hiver - P: Printemps - E : Eté - A : Automne. 
Enfin les performances de reproduction antérieures, nés totaux, nés vivants, nombre de sevrés, des femelles ayant obtenu des portées avant laparatomie ont également été recueillies.

\section{2. - Analyses effectuées}

L'étude porte d'une part, sur la fertilité (nombre de femelles ovulant et gestant par rapport au nombre saillies, quel que soit le nombre de présentations pour les obtenir), d'autre part, sur la prolificité (tailles de portée) et ses composantes : nombre d'ovules pondus, de sites d'implantation et d'embryons. Un estimation de la mortalité embryonnaire avant implantation $\frac{\text { (corps jaunes - sites) }}{\text { corps jaunes }}$ et après implantation (sites - embryons) sites

Les variables continues ont été analysées suivant la méthode des moindres carrés (HARVEY, 1975) avec test et estimation des effets considérés comme fixés dans un schéma croisé d'analyse de variance. Le modèle s'écrit :

$$
X_{i j k l}=\mu+a_{i}+b_{j}+c_{k}+(a b)_{i j}+(a c)_{i k}+(b c)_{j k}+e_{i j k l}
$$

où $\mathrm{X}_{\mathrm{ijkl1}}$ est la mesure faite sur la lième femelle du ième génotype au jème stade de la reproduction, à la saison $\mathrm{k}$.

Une analyse de covariance a aussi été effectuée sur certaines données selon le modèle suivant d'analyse :

$$
Y_{i j k 1}=\mu+a_{i}+b_{j}+c_{k}+(a b)_{i j}+(a c)_{i k}+\beta X_{i j k 1}+e_{i j k 1}
$$

où $\mathrm{X}_{\mathrm{ijkl}}$ est la mesure faite sur un caractère s'exprimant plus tôt que $\mathrm{Y}_{\mathrm{ijkl}}$ dans le cycle de reproduction de la femelle considérée. Ce modèle permet d'estimer par exemple les effets des différents facteurs, pour le nombre de sites d'implantation linéairement indépendant du nombre d'ovules. Pour les variables tout ou rien, les estimées procèdent de l'utilisation des valeurs observées pour chacune des cellules élémentaires du modèle et les tests d'indépendance des effets sont utilisés par $\chi^{2}$.

\section{Résultats}

Dans cette partie pour les différentes variables étudiées, nous analyserons les résultats, fréquence des événements, valeurs moyennes et estimées des moindres carrés.

\section{1. - La fertilité - Ses composantes}

Le tableau 2 indique les valeurs calculées pour la fréquence des femelles saillies qui ovulent et qui sont gestantes. 63 p. 100 des femelles ayant accepté le mâle ovulent; pour ce critère, les deux génotypes sont identiques. Le seul effet hautement 
significatif $\left(\chi^{2}=5,14\right)$ est celui lié à la parité : les femelles nullipares ovulent plus fréquemment que les autres $(+20$ p. 100). La saison semble légèrement affecter cette fréquence d'ovulation $\left(\chi^{2}=6,41\right)$ : il apparaît un effet dépressif en hiver.

\section{TABleaU 2}

Composantes du taux de mise-bas

Components of litter rate

\begin{tabular}{|c|c|c|c|}
\hline \multirow[b]{2}{*}{ Source de variation } & \multicolumn{2}{|c|}{ Femelles saillies } & \multirow{2}{*}{$\begin{array}{c}\text { Femelles ovulant } \\
\text { Fréquence des femelles } \\
\text { n'ayant aucun site } \\
\text { d'implantation }\end{array}$} \\
\hline & $\begin{array}{l}\text { Fréquence des } \\
\text { femelles ovulant }\end{array}$ & $\begin{array}{l}\text { Fréquence des } \\
\text { femelles gestant }\end{array}$ & \\
\hline $\begin{array}{l}\text { Californien } \ldots \ldots \ldots \\
\text { Néo-Zélandais } \ldots \ldots \ldots\end{array}$ & $\begin{array}{l}0.63 \text { (a) } \\
0.63 \mathrm{a}\end{array}$ & $\begin{array}{l}0.49 \mathrm{a} \\
0.57 \mathrm{a}\end{array}$ & $\begin{array}{l}0.21 \mathrm{a} \\
0.09 \mathrm{~b}\end{array}$ \\
\hline $\begin{array}{c}\text { Nullipares } \quad \ldots \ldots \ldots \\
\text { Primipares } \\
\text { Multipares } \ldots \ldots \ldots \ldots\end{array}$ & $\begin{array}{ll}0.77 \mathrm{a} \\
0.56 \mathrm{~b} \\
0.57 \mathrm{~b}\end{array}$ & $\begin{array}{ll}0.68 & \mathrm{a} \\
0.46 \mathrm{~b} \\
0.47 \mathrm{~b}\end{array}$ & $\begin{array}{ll}0.12 & \mathrm{a} \\
0.17 & \mathrm{a} \\
0.18 & \mathrm{a} \\
\end{array}$ \\
\hline $\begin{array}{l}\text { Hiver } \ldots \ldots \ldots \ldots \\
\text { Printemps } \ldots \ldots \ldots \ldots \\
\text { Eté } \ldots \ldots \ldots \ldots \ldots \\
\text { Automne } \ldots \ldots \ldots \ldots\end{array}$ & $\begin{array}{l}0.51 \mathrm{a} \\
0.65 \mathrm{ab} \\
0.73 \mathrm{~b} \\
0.63 \mathrm{ab}\end{array}$ & $\begin{array}{l}0.40 \mathrm{a} \\
0.55 \mathrm{ab} \\
0.59 \mathrm{~b} \\
0.58 \mathrm{ab} \\
\end{array}$ & $\begin{array}{l}0.22 \mathrm{a} \\
0.16 \mathrm{ab} \\
0.20 \mathrm{ab} \\
0.04 \mathrm{~b}\end{array}$ \\
\hline Total & 0.63 & 0.53 & 0.15 \\
\hline
\end{tabular}

(a) Le classement des valeurs moyennes par cellule a été fait par $\chi^{2}$, le seuil de signification admis étant de 10 p. 100.

The ranging of mean values of each class is done by $\chi^{2}$ test. The level of significant difference is $10 \mathrm{p} .100$.

Parmi les femelles qui ovulent, 15 p. 100 en moyenne ne présentent aucun développement embryonnaire à 12 jours. Les deux souches se comportent un peu différemment $\left(\chi^{2}=3,40\right) .9$ p. 100 des femelles Néo-Zélandaises montrent cette anomalie qui atteint 21 p. 100 chez les femelles Californiennes.

La fréquence des femelles gestantes après saillie (par suite de l'existence de ces lapines ovulant et vides) présente des variations, mineures toutefois, par rapport aux femelles ovulant. Une différence non significative apparaît entre les deux souches à 12 jours : la Californienne présentant un taux de gestation de 49 p. 100 alors qu'il est de 57 p. 100 chez la Néo-Zélandaise. La saison semble légèrement affecter cette fréquence d'ovulation $\left(\chi^{2}=6,41\right)$ : il apparaît un effet dépressif en hiver.

\section{2. - La prolificité}

Nous analyserons l'effet du génotype, de la parité et de la saison sur les deux composantes de la prolificité : nombre d'ovules pondus et viabilité embryonnaire avant de commenter brièvement l'évolution de la taille de portée. 

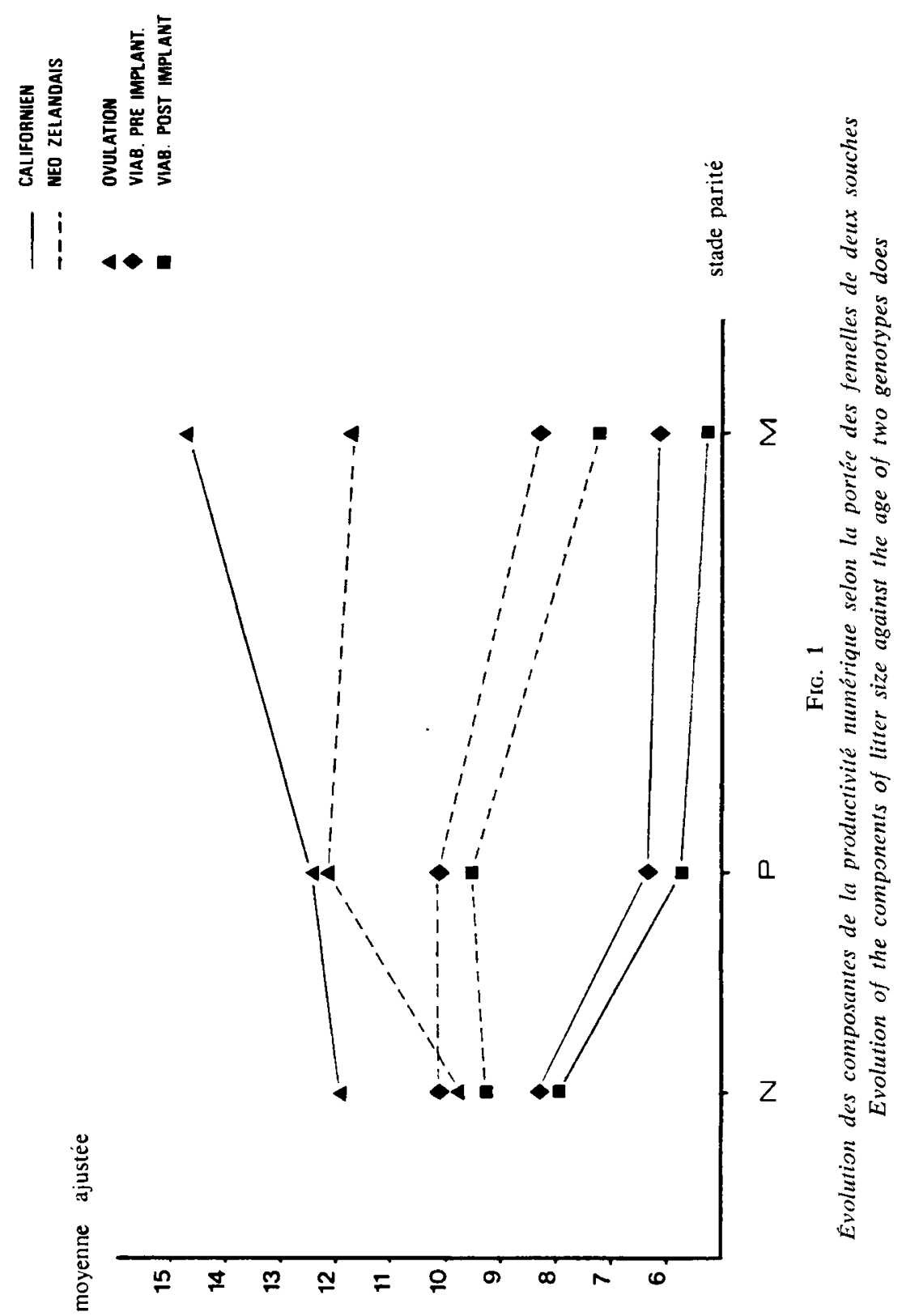


\section{1. - Nombre d'ovules pondus}

L'analyse de variance a été pratiquée sur tous les résultats des femelles ovulant d'une part (112) (tableau 3) et sur ceux des femelles réalisant une gestation d'autre part, (95) (tableau 4). Les résultats étant très comparables dans les deux lots, les commentaires seront faits sur les résultats du premier lot.

Le nombre moyen de corps jaunes est de 12,06. Une différence significative au seuil 1 p. 100, de deux corps jaunes est enregistrée entre les deux génotypes.

L'effet stade est lui aussi hautement significatif avec une supériorité des multipares par rapport aux nullipares de 2,42 corps jaunes indiquant un accroissement du nombre d'ovules pondus en fonction de la parité.

La présence d'une interaction à la limite de la signification (seuil 10 p. 100) entre le génotype et la parité (significative au seuil de 5 p. 100 sur les 95 femelles ayant des sites d'implantation) indique que la progression du nombre d'ovules pondus avec la parité n'est pas la même dans les deux génotypes. La Néo-Zélandaise paraît atteindre plus tôt ses performances maximales les résultats obtenus au stade multipare étant sensiblement équivalents à ceux du stade primipare (fig. 1).

L'effet saison est significatif au seuil 10 p. 100. Il apparaît au printemps un avantage de $+1,12$ ovule par rapport à la moyenne.

\section{2. - Développement embryonnaire}

\section{- Période pré-implantatoire}

- L'analyse de la mortalité avant implantation sera tout d'abord faite sur toutes les femelles ovulant. Cette population fournit globalement un pool de corps jaunes et de sites dont l'écart indique l'importance du nombre d'ovules peu ou pas développés, quelles qu'en soient les causes. Nous la qualifierons de «mortalité totale» au sens large.

Le tableau 3 indique que les différences enregistrées entre génotypes sur le nombre d'ovules pondus s'estompent lorsqu'on s'intéresse au nombre de sites d'implantation, ce qui laisse présager une mortalité pré-implantatoire différente (40 p. 100 chez la Californienne et 21 p. $100 \mathrm{chez}$ la Néo-Zélandaise). L'analyse de covariance qui permet d'estimer plus précisément cette mortalité, à nombre d'ovules constant, confirme cette différence entre génotypes (- 1,28 site d'implantation chez la Californienne contre $+1,28 \mathrm{chez}$ la Néo-Zélandaise, valeurs significativement différentes au seuil de 1 p. 100).

L'effet parité montre un accroissement de la mortalité qui passe de 24 p. 100 chez les nullipares à 38 p. 100 chez les multipares. L'analyse de covariance à nombre de corps jaunes constant, confirme cette tendance (significative au seuil de 10 p. 100) indiquant une implantation défectueuse chez les multipares $(-1,11$ site) par rapport aux nullipares $(+0,89)$.

Un effet saisonnier paraît se manifester bien que non significatif : le printemps et l'aütomne assureraient une meilleure viabilité.

- Après cette première analyse, il nous paraît important de préciser la provenance de cette mortalité totale en distinguant les femelles qui, à 12 jours, présentent ou non des signes de développement embryonnaire, les mécanismes physiologiques respon- 


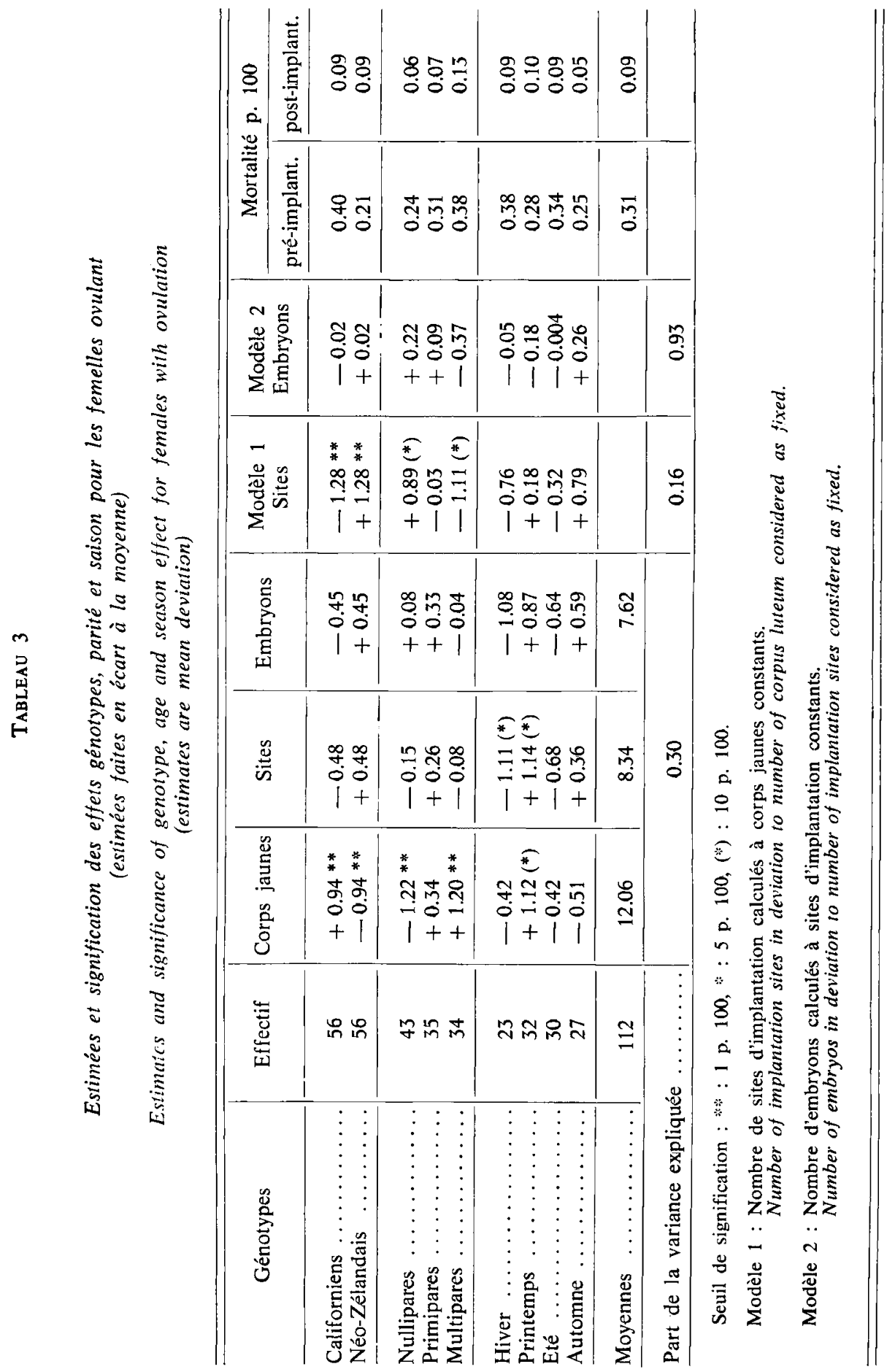


LAPINE REPRODUCTIVE : GENOTYPE, AGE, SAISON

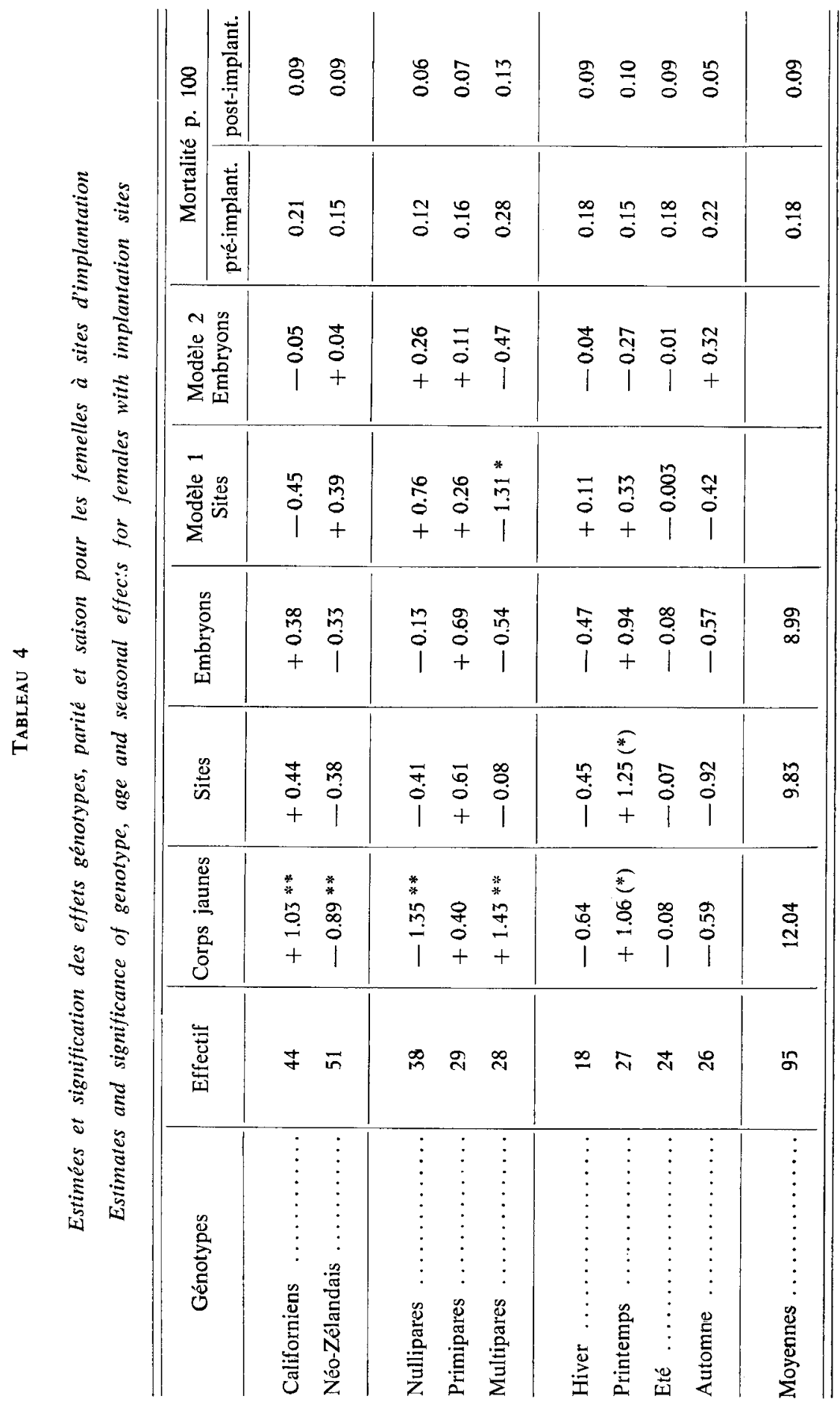


sables de ces deux situations étant différents (Hafez, 1965). C'est pourquoi, nous analyserons également sur les 95 femelles ovulant et gestant, l'importance de la «mortalité partielle» (tableau 4).

L'interprétation des résultats n'est pas fondamentalement changée pour les deux premiers critères d'étude. La différence de mortalité entre les deux génotypes subsiste (15 p. 100 pour la Néo-Zélandaise et 21 p. 100 pour la Californienne).

L'augmentation des pertes embryonnaires en fonction de la parité apparaît plus clairement (significatif au seuil de 5 p. 100).

Aucune interaction significative n'apparaît entre le génotype et la parité. Cependant, les deux souches se comportent différemment, la Néo-Zélandaise présentant un accroissement considérable des pertes au cours de la quatrième gestation (6 p. 100, 11 p. 100 et 30 p. 100 aux différentes parités étudiées), alors que l'augmentation est régulière chez la Californienne (17 p. 100, 22 p. 100 et 26 p. 100). L'évolution du nombre de sites d'implantation qui en résulte est indiquée dans la fig. 1.

L'effet saison n'est pas significatif mais la tendance observée indique que la viabilité pré-implantatoire et la quantité d'ovules pondus subissent des influences saisonnières similaires, ce qui accentue l'effet favorable au printemps et défavorable en automne sur le nombre d'implants.

\section{- Période post-implantatoire}

Lorsqu'une femelle présente des sites d'implantation à 12 jours, il est rare semblet-il qu'une mortalité totale survienne : 1 femelle sur 95 présente un tel événement. La fréquence de la mortalité fœtale partielle (tableau 4) s'élève à 9 p. 100 à 12 jours dans les deux génotypes et ne présente pas de variation particulière si ce n'est une légère augmentation avec la parité.

La moyenne des embryons obtenus nous conduit à l'estimation de la future taille de portée.

\section{3. - Taille de portée}

La prolificité résulte d'un équilibre entre deux composantes : le nombre d'ovules pondus et leur viabilité après fécondation qui évoluent indépendamment.

\section{- Prolificité potentielle (nombre d'embryons à 12 jours de gestation)}

L'étude des composantes nous apprend que l'écart moyen initial de deux ovules entre les deux génotypes se trouve estompé par une mortalité inverse qui ne laisse à la femelle Californienne qu'une supériorité non significative de 0,7 embryon à 12 jours (tableaux 4 et 5).

Au niveau de l'âge, l'augmentation du nombre d'ovules pondus et la diminution de la viabilité embryonnaire donne lieu à un équilibre en faveur, dans notre expérience, des primipares (ou multipares avant la $4^{\mathrm{e}}$ portée).

Un effet saison paraît subsister : la taille de portée est la plus faible en automne et la plus élevée au printemps. 


\section{TABleau 5}

Comparaison des prolificités observées et potentielles

Comparison of observed and potential litter sizes

\begin{tabular}{|c|c|c|c|c|c|c|}
\hline & \multicolumn{2}{|c|}{ Prolificité potentielle } & \multicolumn{4}{|c|}{ Prolificité post-natale } \\
\hline & $\begin{array}{l}\text { Nombre } \\
\text { femelles }\end{array}$ & $\begin{array}{l}\text { Nombre } \\
\text { embryons } \\
\text { à } 12 \text { jours }\end{array}$ & $\begin{array}{l}\text { Nombre } \\
\text { de portées }\end{array}$ & $\begin{array}{l}\text { Nés } \\
\text { totaux }\end{array}$ & $\begin{array}{l}\text { Nés } \\
\text { vivants }\end{array}$ & $\begin{array}{l}\text { Nés } \\
\text { sevrés }\end{array}$ \\
\hline Californiens & 18 & 9,37 & 127 & 8,76 & 8,05 & 6,95 \\
\hline Néo-Zélandais & 20 & 8,66 & 115 & 7,35 & 6,95 & 5,99 \\
\hline $\begin{array}{c}\text { Nullipares } \ldots \\
1^{\text {re }} \text { portée }\end{array}$ & 38 & 8,86 & 60 & 7,80 & 7,27 & 6,23 \\
\hline $\begin{array}{c}\text { Primipares } \\
2^{\mathrm{e}} \text { portée }\end{array}$ & 29 & 9,68 & 61 & 8,07 & 7,66 & 6,66 \\
\hline $\begin{array}{c}\text { Multipares } \ldots \ldots \\
3^{e}-4^{\mathrm{e}} \text { portées }\end{array}$ & 28 & 8,45 & 59 & 9,06 & 8,58 & 7,56 \\
\hline
\end{tabular}

\section{- Prolificité à la naissance}

Les performances générales des femelles durant toute leur carrière de reproductrice (portée 1 des primipares, portées 1,2 et 3 des multipares) sont mentionnées dans le tableau 5. La souche Californienne présente en moyenne des performances supérieures à la souche Néo-Zélandaise à la naissance $(1,4$ lapereau) qui s'estompent légèrement au sevrage (1 lapereau).

Les tailles de portées successives ont été estimées sur les femelles multipares. Une augmentation de 1,2 lapereau à la naissance est constatée entre la portée 1 et 3 , avantage qui se maintient au sevrage. L'effet saison n'a pu être pris en compte du fait de l'étalement des naissances des différentes portées dans le temps.

\section{Discussion}

\section{La fertilité}

De nombreux facteurs peuvent influer sur l'aptitude à ovuler : le génotype, l'intervalle saillie - mise-bas, la lactation, la saison.

- Les deux souches étudiées présentent une aptitude à ovuler comparable mais nous avons vu, lors de travaux antérieurs (Hulor \& Matheron, 1979), que certains génotypes, le Petit Russe en particulier, utilisé en race pure ou introduit en croisement dans différentes souches (notamment la Néo-Zélandaise et la Californienne) conduisait à un pourcentage élevé de femelles ovulant de l'ordre de 80 à 85 p. 100. 
- Nous avons pratiqué les saillies pour les femelles primipares et multipares en état de lactation 10 jours après la mise-bas antérieure. Si selon PrUD'HON et al. (1969), un tel délai est satisfaisant (testé sur le taux de mise-bas), selon FoxcrofT \& HasNAIN (1973) il est à peine favorable et la lactation contribuerait par une compétition entre besoins d'entretien et de production, à diminuer son intérêt (testé sur la fréquence des femelles ovulant et valable pour certain génotype, Large Albino). Ceci pourrait éventuellement expliquer la supériorité des femelles nullipares (donc non allaitantes) de notre expérience pour l'aptitude à répondre positivement à la saillie.

- La saison, pour beaucoup d'auteurs, a un effet défavorable en automne qui abaisse la fréquence d'ovulation (Farrel et al., 1968; Selme \& Prud'hon, 1973), et le taux de mise-bas. Pour les uns cet effet commence en été et serait lié à un effet défavorable des hautes températures (SitTman et al., 1964). Pour d'autres, il se situerait plus en automne-hiver et dépendrait essentiellement de la durée d'éclairement : WALTER et al. (1968) augmentent le pourcentage de femelles en ostrus en soumettant les animaux à 16 heures de lumière au lieu de 8 et 12 heures. FARrel et al. (1968) constatent un faible taux de femelles qui ovulent en jours courts consécutivement à l'absence de synthèse ou de décharge de LH. A la limite, dans un environnement parfaitement contrôlé en ce qui concerne la température et la lumière, il n'y aurait plus d'effet saisonnier significatif (Torres et al., 1977). Dans notre élevage où les conditions du milieu sont en partie maîtrisées, nous aurions peu de variations dans l'aptitude à ovuler (effet ponctuel de l'environnement) et à gester (effet cumulatif du milieu) sur l'ensemble des deux souches, si ce n'est l'effet défavorable de la saison d'hiver. Cette période étant celle du démarrage de l'expérience, nous pensons en définitive qu'il s'agit peut-être plus d'un stress lié à une mise en route récente des contrôles qu'à un véritable effet saisonnier.

- La disparité qui existe entre le pourcentage de femelles ovulant et gestant (15 p. 100 des femelles qui ovulent sont vides) est mentionnée par d'autres auteurs : 7 p. 100 selon Adams (1960), 10 p. 100 selon Selme \& Prud'hon (1973) chez le Lapin, 7 p. 100 par HARPER (1964) chez le Rat, 6 à 20 p. 100 selon Edwards \& Fowler (1959) chez la Souris. L'origine en est mal déterminée : il est possible d'invoquer des événements tels que : un sperme défectueux, l'absence de fécondation ou une mortalité précoce due à une stérilité passagère en relation avec un déséquilibre endocrinien ou des conditions pathologiques (HAFEZ, 1965). Un tel événement paraît plus fréquent en souche Californienne, 21 p. 100, qu'en souche Néo-Zélandaise, 9 p. 100, fait déjà observé en saillie post partum (Hulot \& MATHeron, 1979 : 22 et 5 p. 100 respectivement).

\section{La prolificité}

\section{L'ovulation}

Nos travaux antérieurs (Hulot \& Matheron, 1979) avaient montré l'écart qui existait (près de 2 ovules) en faveur du Californien par rapport au Néo-Zélandais. Les estimées de cette expérience confirment cette différence et contribuent ainsi à caractériser sur ce point les souches que nous employons. Lorsque la femelle vieillit, le nombre d'ovules pondus semble augmenter; telle est du moins la conclusion que nous pouvons tirer de cette étude. Une part de cet accroissement pourrait être dû au 
phénomène d'aillaitement : selon Foxcroft \& HASNAIN (1973) les moyennes de corps jaunes diffèrent significativement entre femelles primipares (Large Albino) allaitantes et non allaitantes. Adams (1970) note une très faible variation de ce paramètre au cours des 11 premières portées. Cependant chez d'autres espèces polytoques, l'accroissement du nombre d'ovules pondus au cours de la vie de l'animal paraît un phénomène assez général : chez la Souris, MAC Dowell \& LoRd (1925) notent une augmentation rapide au début de la vie reproductive (3 portées) puis un plateau durable. Chez la truie, HAMmond dès 1914, affirme que les animaux âgés pondent plus d'ovules que les jeunes, ce qui paraît confirmé par Perry (1954) ; 13,6 corps jaunes chez les nullipares; 24,4 chez les multipares ayant réalisé 7 portées et plus.

La saison d'accouplement paraît jouer sur le nombre moyen d'ovules pondus (1,7 corps jaunes d'écart entre hiver et printemps). Les résultats enregistrés dans la bibliographie sont très divers allant de la description de variations mineures (HAFEz, 1964) à celle d'écarts plus marqués (SElme \& PRUD'Hon, $1973:+2,8$ ovules au printemps par rapport à l'automne), et parfois même très significatifs (PILAWSKI, 1969, $+3,9$ ovules aux saisons précédemment citées). Ces différences avec les autres auteurs, comme pour la fertilité, sont peut-être liées en partie aux conditions d'élevage, d'utilisation de programme lumineux et régulation de température qui tendent à atténuer l'influence naturelle de la saison sur les performances de reproduction des animaux (MYKYTOWYCZ \& Fullagar, 1973).

\section{Le développement embryonnaire}

- Nos résultats en souche pure confirment ce que nous avions précédemment trouvé sur ces mêmes souches (utilisées en croisement), à savoir un pourcentage de mortalité pré-implantatoire globale élevé chez la Californienne (32,73 et 40 p. 100) et plus réduit chez la Néo-Zélandaise $(15,05$ et 21 p. 100). Les valeurs de notre expérience un peu plus importantes dans les deux génotypes peuvent s'expliquer par l'absence d'un hétérosis favorable à la viabilité. Selme \& Prud'hon (1973), sur des femelles communes et Néo-Zélandaises, obtiennent des pourcentages de mortalité préimplantatoires variant de 22 à 33 p. 100, FoxCroft \& HASNAIN (1973), les évaluent à 30 p. 100 .

Les pertes partielles sont également plus élevées chez la femelle Californienne que chez la femelle Néo-Zélandaise, ce qui confirme les qualités maternelles défavorables de cette souche ou une fréquence plus élevée de produits létaux. Les estimations faites par d'autres auteurs sont généralement un peu plus faibles $(9,7$ p. 100, AdAMS, $1960 ; 12-13$ p. 100, Foxcroft \& Hasnain, $1973 ; 9$ à 13 p. 100 chez le Rat, HARPER, 1964). La mortalité post-implantatoire ne discrimine par les génotypes à 12 jours, elle est comparable aux observations de ADAMS qui l'estime à environ 7 p. 100.

- En ce qui concerne l'augmentation de la mortalité pré-implantatoire et post-implantatoire en fonction de la parité, il convient d'être nuancé. En effet, l'allaitement est considéré généralement (HAFEZ \& IsHibashi, 1965 ; HARNed \& CASIDA, 1969) comme un facteur défavorable pour le déroulement d'une nouvelle gestation simultanée, notamment en ce qui concerne la survie de l'embryon et peut donc interférer avec l'effet vieillissement. Ceci expliquerait la meilleure viabilité des femelles 
nullipares non allaitantes. Cependant, entre primipares et multipares, dans des conditions comparables (intervalle mise-bas - saillie identique, allaitement), la mortalité progresse sensiblement.

Cette augmentation de la mortalité prénatale en fonction de l'âge maternel ou de la parité paraît un phénomène assez général chez les espèces polytoques, les pertes étant plus ou moins accentuées avant ou après l'implantation selon les auteurs et les espèces. Adams (1970) sur le Lapin trouve que seule la mortalité post-implantatoire s'accentue entre la troisième et septième portée au-delà de laquelle cette progression continue. Perry (1954) chez la Truie note une prédominance de la mortalité dans la première partie de la gestation et au moment de l'implantation. FinN (1963) chez la Souris, Thorneycroft \& Sordewall (1969) chez le Hamster, Talbert (1968) chez divers rongeurs constatent une baisse de viabilité avec l'âge. Les causes de ce phénomène sont très discutées : outre la lactation, l'augmentation du nombre d'ovules pondus avec la parité (PERRY, 1954) contribuerait à accroître proportionnellement les pertes embryonnaires, ce qui n'est pas notre cas puisque nous avons constaté une progression de la mortalité à nombre d'ovules constants.

Il est possible que dans certains cas, la qualité de l'ovocyte se détériore, entrâ̂nant des anomalies de fécondation ou un arrêt précoce de la croissance (Adams, 1970). La déficience hormonale a été également évoquée, cependant, chez la Lapine, la fonction endocrinienne de l'ovaire paraît bien maintenue (ADAMS, 1970; LARson et al., 1973). Il semblerait selon la plupart des auteurs (BIGGERs et al., 1962 ; FinN, 1963) qu'il s'agirait essentiellement d'un changement structural des voies génitales, se manifestant par une vascularisation déficiente, un accroissement du contenu de l'utérus en collagène, qui pourrait abaisser le taux d'implantation. $\mathrm{Ce}$ vieillissement serait d'ordre chronologique, dû plus à l'âge qu'aux gestations successives : FinN (1963) note que des femelles gardées vierges pendant 9 mois puis accouplées ont des performances de reproduction comparables à celles de souris du même âge mise en reproduction continue.

\section{Taille de portée}

Qu'il s'agisse de l'estimation de la taille de portée potentielle à 12 jours ou de la taille de portée à la naissance, la souche Californienne présente en moyenne des performances supérieures à la souche Néo-Zélandaise à la naissance (1,5 lapereau) qui s'estompent légèrement au sevrage (1 lapereau). Cette amélioration serait liée si on se réfère à des travaux antérieurs, aux bonnes qualités maternelles de la Néo-Zélandaise. Ce fait est un peu surprenant car au cours des diverses expérimentations, menées jusqu'alors, la prolificité de la Néo-Zélandaise est sensiblement égale, 8,04 CAL et 8,09 NZ (Hulot \& Matheron, 1979) - 7,12 CAL. 7,03 NZ (Matheron, données non publiées), ou même, supérieure à celle de la Californienne, 7,95 NZ - 7,75 CAL. (Poujardieu, Vrillon, 1973).

Malgré l'évolution inverse des composantes de la prolificité, le résultat taille de portée tend à augmenter. Comparé aux résultats de taille de portée potentielle, il semblerait y avoir une prolificité maximale en portée 3 qui décroîtrait dès la portée 4. Ces résultats ne sont pas en opposition avec la littérature mais globalement la prolificité semble atteindre progressivement un plateau (au cours de 3 ou 4 portées) 
et ne décroître que plus tard. (BIGGERs et al., 1962; FinN, 1963, chez la Souris ; INGRAM et al., 1958, chez le Rat ; LARSON et al., 1973, chez le Lapin). De précédents résultats obtenus sur l'ensemble des 3 races étudiées dans notre élevage (Petite Russe, Néo-Zélandaise et Californienne) indiquent que la prolificité atteint son maximum à la quatrième ou cinquième portée (MATHERoN \& RouviER, 1978).

Quant à l'effet saison, estimé sur la taille de portée potentielle, il semble que dans des conditions d'éclairement et de température relativement standardisées, il paraisse subsister : la taille de portée est la plus faible en automne, ce qui va dans le sens des études de SitTman et al. (1964) et la plus élevée en mai, plus tardivement que les observations du précédent auteur (février-mars).

\section{Conclusions}

Parmi les éléments énoncés et discutés, il nous paraît intéressant de mettre en relief certains points qui pourraient caractériser «le Lapin» en général, parallèlement à d'autres espèces et, d'en tirer une certaine connaissance pour une meilleure utilisation des mécanismes physiologiques conduisant à la mise-bas. Nous mettrons ensuite l'accent sur les différences d'ordre génétique et les réflexions qu'elles suscitent.

L'ensemble de nos résultats met en évidence un phénomène antagoniste entre l'évolution de la ponte ovulaire et celle de la capacité implantatoire chez une femelle au fur et à mesure que son âge augmente. Cette règle paraît très générale chez les espèces polytoques. Il semble en effet que le potentiel ovulatoire de l'ovaire ne soit pas le principal responsable de la chute de prolificité. De nombreux auteurs notent la présence durable dans l'ovaire de follicules après cessation de la vie reproductive (Mandl \& Shelton, 1959). Par contre, la mortalité embryonnaire s'accroît inéluctablement. Du fait de cette évolution, l'effectif à la naissance, critère synthétique, ne rendrait pas compte du même phénomène selon le numéro de portée examinée : en première mise-bas, le nombre de nés serait plutôt limité par le nombre d'ovules pondus, au cours des gestations ultérieures c'est la mortalité embryonnaire qui, en définitive, conditionnerait la prolificité. Cette hypothèse confrontée aux résultats des expériences de sélection réalisées notamment chez la Souris (FALCONER, 1971 ; BATEMAN, 1966 ; BRADFORD, 1966 ; JOAKIMSEN \& BAKER, 1977; BAKKER et al., 1978 ; EISEN, 1978) paraît plausible. Ces auteurs, qui ont pratiqué la sélection sur les performances de reproduction mesurées en portée 1, constatent en effet, qu'elle conduit à une augmentation du nombre d'ovules pondus (Matheron \& Mauléon, 1979). Bien que ce résultat ne soit pas surprenant puisque ce caractère est plus héritable que la viabilité, le fait que la portée 1 reflète essentiellement le niveau d'ovulation doit contribuer à renforcer l'impact de la sélection sur ce caractère. On peut alors se demander ce que seraient les résultats si ces expériences étaient pratiquées par exemple sur des femelles au sommet de leur carrière (quatrième - cinquième portée) ou $a$ fortiori, sur l'ensemble de la carrière d'une femelle, lorsque les performances d'ovulation sont masquées par les problèmes de développement embryonnaire. L'évolution de l'expressivité des caractères au cours de la vie de l'animal pose le problème de l'optimisation des méthodes de sélection pour assurer un meilleur progrès sur le «meilleur critère » de sélection qu'il faudrait sans doute aborder au vue d'expérimentations plus fines. 


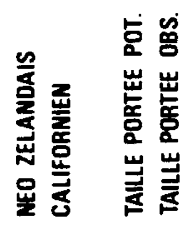
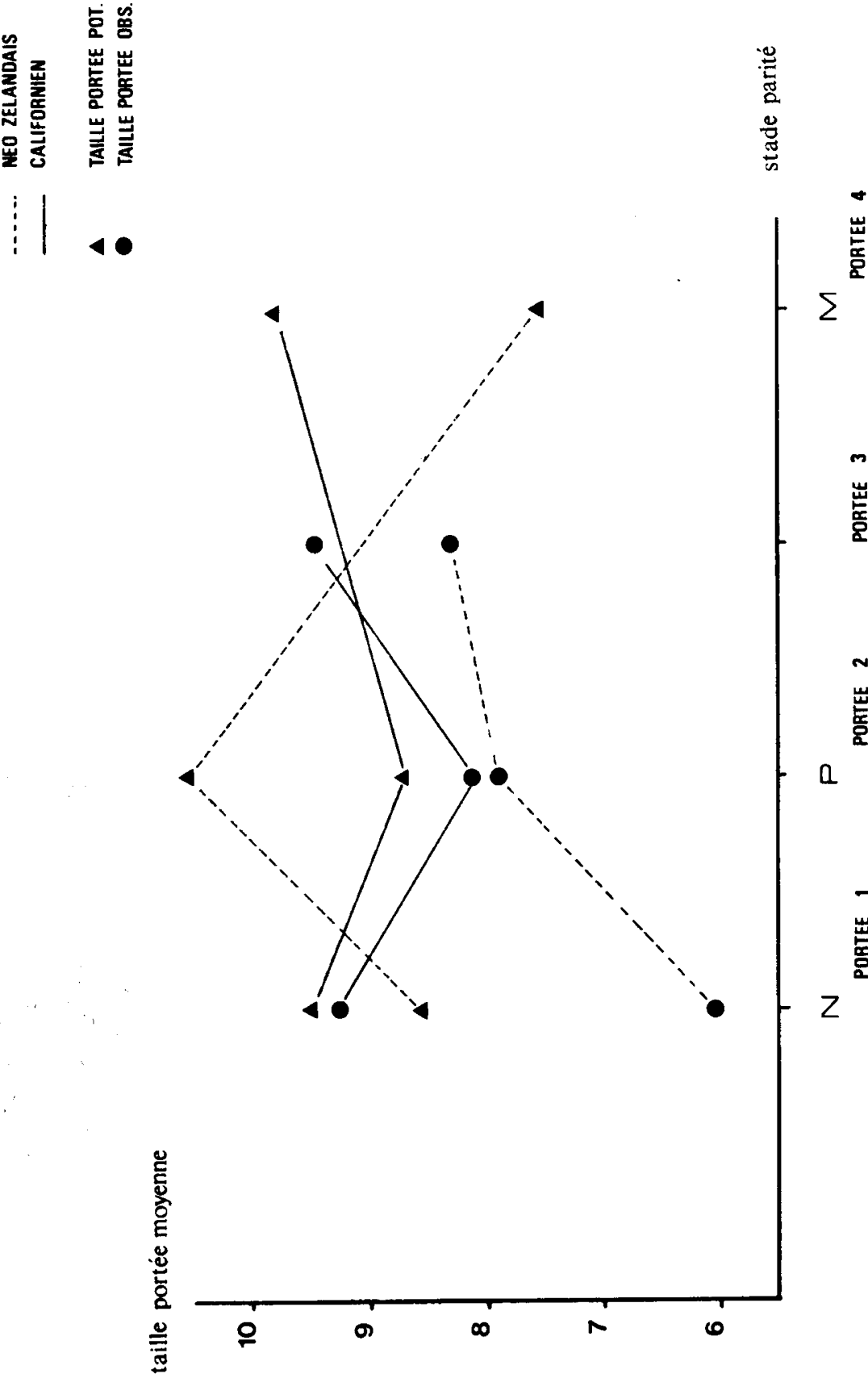
Si nos résultats dans leur ensemble aboutissent à une description claire de la cinétique des composantes de la reproduction, au niveau de chacune des souches, des différences d'évolution apparaissent (interaction génotype-parité). La figure 1 montre à partir des valeurs ajustées par analyse de variance pour les deux génotypes, l'évolution observée du nombre d'ovules pondus, des viabilités pré et post-implantatoires : la Néo-Zélandaise paraît atteindre plus tôt que la Californienne ses performances maximales d'ovulation, par contre, la dégradation du milieu utérin est plus tardive. $\mathrm{Si}$ nos échantillons reflètent bien les performances moyennes des souches, nous sommes alors conduits à penser que des différences de maturité sexuelle pourraient exister entre les deux génotypes et que, de plus, la précocité de chaque composante serait indépendante : une souche pourrait être à la fois précoce pour l'ovulation et tardive pour la mortalité. Nous resterons toutefois réservés sur ce schéma auquel aboutissent nos résultats expérimentaux ayant déjà noté que la comparaison NéoZélandais - Californien en ce qui concerne la prolificité à la naissance était curieusement en légère défaveur de la Néo-Zélandaise. S'agit-il d'une ovulation défectueuse ou d'une mortalité excessive (ou les deux) qui fausserait le stade multipare et donc l'évolution finale du graphique, il serait bon de le confirmer.

Enfin, se pose le problème du choix du stade optimal pour caractériser un génotype. La fig. 2 illustrant les tailles de portées observées à la naissance ou potentielles (12 jours de gestation) indique que le stade primipare est le plus confus notamment chez la Californienne où les performances sont inférieures à celles des nullipares. II apparaît comme un stade de «crise » entraînée par une évolution différente des deux composantes dans chacune des souches qui aboutit à une absence de différence significative entre elles au niveau du nombre total de jeunes nés et de l'ovulation. Le stade nullipare, paraît en définitive celui où s'expriment assez clairement les différences génétiques, ovulation et mortalité embryonnaire reflétant déjà les caractères généraux de la souche : bonne qualité ovarienne de la Californienne et meilleur environnement utérin de la Néo-Zélandaise en général. Le premier critère s'affirme toujours plus à chaque expérimentation et l'étude des populations folliculaires entreprises dans le même cadre expérimental apportera certainement des indications sur l'origine de ces écarts. Au niveau de la mortalité in-utero, des tendances se dessinent mais plus confuses, la variabilité observée étant due à des origines plus complexes et moins génétiques (les modèles interprétatifs utilisés expliquent seulement 16 p. 100 de la variance pour les sites contre 30 p. 100 pour l'ovulation). En outre, une meilleure définition de la mortalité embryonnaire pré-implantatoire reste nécessaire — cas des femelles ne présentant aucune trace d'implantation tout en ayant ovulé - et une connaissance plus complète de la mortalité post-implantatoire serait indispensable. Lors d'expérimentations ultérieures et complémentaires sur ce thème, il serait judicieux d'étudier l'effet «vieillissement» indépendamment de l'effet lactation qui, non seulement au niveau de la mortalité mais aussi de l'ovulation, masque sans doute certaines réalités.

Reçu pour publication en mai 1981.

\section{Remerciements}

Nous remercions tout particulièrement G. Catriau et R. Duzert, pour leur collaboration technique. 


\begin{abstract}
Summary
The influence of genotype, age and season on the reproduction components in the female rabbit
\end{abstract}

A comparison between 120 Californian and Neo-Zealand white, females rabbits selected at the Animal Genetic Improvement Station of Toulouse, slaughtered after mating at nulliparous, primiparous and multiparous stage (in fourth gestation) and at the four seasons gives us the following results :

- a Californian doe, whatever are the female age and the season of the mesure, shed two ova more than a New-Zealand white doe; types ;

- the study of pre-implantation losses gives a reverse ranking for these two geno-

- the aging effect on a female is increasing the number of ova shed and dimishing its « uterine capacity»;

- a primiparous doe has the greatest number of embryos because of a favourable conjunction of such opposite mecanisms;

- the study of the seasonal effects shows differential results against the trait considered.

\title{
Références bibliographiques
}

Adams C.E., 1960. Studies on prenatal mortality in the rabbit, Oryctolagus Cuniculus : the amount and distribution of loss before and after implantation. J. Endocrinol., 19, 325-344.

ADAmS C.E., 1970. Ageing and reproduction in the female mammal with particular reference to the rabbit. J. Reprod. Fert., suppl., 12, 1-16.

Bateman N., 1966. Ovulation and post ovulational losses in strains of mice selected from large and small litters. Genet. Res., 8, 229-241.

Bakker H., Wallingh H., Politiek R.D., 1978. Reproduction and body weight of mice after long term selection for litter size. J. anim. Sci., 46, 1572-1580.

Biggers J.D., Finn C.A., Mc Laren Anne, 1962. Long-terme reproductive performance of female mice. II variation of litter size with parity. J. Reprod. Fertil., 3, 313-330.

BradFORD G.E., 1966. Effects selecting for reproductive characteristics in mice. Genetics, 54, 324.

Edwards R.G., Fowler R.E., 1959. Fetal mortality in adult mice after super ovulation with gonadotrophines. J. exp. Zool., 41, 229.

EISEN E.J., 1978. Single trait and antagonistics index selection for litter size and body weight in mice. Genetics, 88, 781-811.

Falconer D.S., 1971. Improvment of litter size in a strain of mice at a selection limit. Genet. Res., 17, 215-235.

Farrell G., Powers D., Otani, 1968. Inhibition of ovulation in the rabbit : seasonal variation and the effect of indoles. Endocrinology, 83, 599-603.

FinN C.A., 1963. Reproductive capacity and litter size in mice : effect of age and environment. J. Reprod. Fert., 6, 205-214.

Fox R.R., 1980. The rabbit and research on Ageing. Exp. Ageing Res., 6, 235-248.

FOXCROFT G.R., HaSNAIN H., 1973. Effects of suckling and time to mating after parturition on reproduction in the domestic rabbit. J. Reprod. Fert., 33, 367-377. 
HAFEZ E.S.E., 1964. Seasonal fluctuations in ovulation rate and superovulatory response of the domestic rabbit (Oryctolagus Cuniculus). Acta zool. Stockholm, 45, 123-131.

HAfEZ E.S.E., 1965. Quantitative aspects of implantation embryonic survival and fetal development. Int. J. Fert., 10, 235-251.

Hafez E.S.E., Ishibashi I., 1965. Effect of lactating and age at first breeding on size and survival of rabbit blastocysts. Int. J. Fert., 10, 47-55.

Hammond J., 1914. On some factors controlling fertility in domestic animals. J. agric. Sci., 6, 263-277.

Harned M.A., Casida L.E., 1969. Some post partum reproductive phenomena in the domestic rabbit. J. anim. Sci., 28, 785-788.

HARPER M.J.K., 1964. Observations on amount and distribution of prenatal mortality in a strain of albino rats. J. Reprod. Fert., 7, 185-209.

HARVEY W.R., 1975. Least squares analysis of data with unequal subclass number. Agricultural Research Service. VS. Department of Agriculture.

Hulot F., Matheron G., 1979. Analyse des variations génétiques entre trois races de lapins de la taille de portée et de ses composantes biologiques en saillie post partum. Ann. Génét. Sél. anim., 11, 53-77.

Ingram D.L., Mande Anita M., Zuckerman S., 1958. The influence of age on litter size. J. Endocrinol., 17, 280-285.

Johimsen O., Baker R.L., 1977. Selection for litter size in mice. Acta Agric. scand., 27, 301-318.

Larson L.L., Spilman C.H., Dunn H.A., Foote R.H., 1973. Reproductive efficiency in aged female rabbits given supplemental progesterone and oestradiol. J. Reprod. Fert., 33, 31-38.

Mande A.M., Shelton M., 1959. A quantitative study of cocytes in young and old nulliparous laboratory rats. J. Endocrinol., 18, 444-450.

Matheron G., Mauléon P., 1979. Mise en évidence des actions conjointes des effets directs maternels et grands maternels sur la taille de portée. Bull. Techn. Dép. Génét. anim. (Inst. Rech. Agron. Fr.), $\mathrm{n}^{\circ}$ 29-30, 232-274.

Matheron G., Poujardieu B., 1976. Heterosis pour quelques caractères de reproduction chez le Lapin. Analyse de plans de croisement. Bull. Techn. Dép. Génét. anim., 24, 69-77.

Matheron G., Rouvier R., 1978. Etude de la variation génétique dans le croisement simple entre 6 races de lapins pour les caractères de prolificité taille et poids de portée au sevrage. $2^{\text {es }}$ journées de la recherche cunicole. Communication $n^{\circ} 22,4$ et 5 avril, Toulouse.

MC Dowell E.C., LoRd E.M., 1925. The number of corpora lutea in successive Mouse pregnancy. Anat. Rec., 31, 131.

MYrkytowycz R., Fullagar P.J., 1973. Effect of social environment on reproduction in the rabbit. J. Reprod. Fert., suppl., 19, 503-522.

PERRY J.S., 1954. Fecundity and embryonic mortality in pigs. J. Embryol. exp. Morph., 2, 308-322.

PILAWSKY Z., 1969. Seasonal variations of ovulation response time after copulation in rabbits. Folia biol. Krakow, 17, 211-217.

Poujardieu B., VRillon J.L., 1973. Variation de la productivité numérique au sevrage et de ses composantes entre génotypes de lapines croisées et de race pure. Journ. Recher. avic. et cunic., décembre 1973, 89-93, ITAVI.

Prud'hon M., Rouvier R., Cael J., Bel L., 1969. Influence de l'intervalle entre la parturition et la saillie sur la fertilité et la prolificité des lapins. Ann. Zootech., 18, 317-329.

Selme, Prud'hon M., 1973. Comparaison, au cours de différents saisons, des taux d'ovulation, d'implantation et de survie embryonnaire chez des lapines allaitantes saillies à l'œstrus post partum et chez des lapines témoins. Journ. Rech. avicole et cunicole, décembre 1973, pp. 55-58. 
Sittmann D.B., Rollins W.C., Sittmann K., Casady R.R., 1964. Seasonal variation on reproductive traits of the New-Zealand white rabbits. J. Reprod. Fert., 8, 29-37.

TALbert G., 1968. Effect of maternal age on reproductive capacity. Ann. J. Obstet. Gynecol., 102, 451.

Thorneycroft, Soderwall, 1969. The nature of the litter size loss in senescent hamsters. Anat. rec., 165, 343.

Torres S., Gerard M., Thibault C., 1977. Fertility factors in lactating rabbits mated 24 hours and 25 days after parturition. Ann. Biol. anim. Biochim. Biophys., 17, 63-69.

Walter M.R., Martinet L., Moret B., Thibault C., 1968. Régulation photopériodique de l'activité sexuelle chez le lapin mâle et femelle. Arch. Anat. Histol. Embryol., 51, 773-780. 